\title{
Expression of the envelope antigen F1 of Yersinia pestis is mediated by the product of cafl $M$ gene having homology with the chaperone protein PapD of Escherichia coli
}

\author{
E.E. Galyov ${ }^{1}$, A.V. Karlishev ${ }^{2}$, T.V. Chernovskaya ${ }^{1}$, D.A. Dolgikh ${ }^{1}$, O.Yu. Smirnov ${ }^{1}$, K.I. Volkovoy ${ }^{2}$, \\ V.M. Abramov' and V.P. Zav'yalov' \\ ${ }^{1}$ Institute of Immunology, 142380 Lyubuchany, Moscow Region, USSR and ${ }^{2}$ All-Union Research Institute of Applied Microbiology, \\ 142279 Obolensk, Moscow Region, USSR
}

Received 23 April 1991

The effective synthesis of the envelope antigen FI of $Y$. pestis in $E$. coli HB101 is mediated by the expression of the caflM gene. This gene was sequenced, and the protein encoded was found to have a significant homology with the chaperone protein PapD of uropathogenic $E$. coli. The data presented allow one to suppose CaflM and PapD proteins perform similar functions in the biogenesis of the $Y$. pestis capsule and $E$. coli P-pili, respectively.

Chaperone protein; Nucleotide sequence; Envelope antigen expression; Yersinia pestis

\section{INTRODUCTION}

Most strains of $Y$. pestis (causative agent of plague) possess the virulence plasmid pFra (110 kb in size) which mediates the expression of the fraction 1 envelope antigen at $37^{\circ} \mathrm{C}[1]$. The envelope is associated with virulence, and the protein $F 1$ is able to induce protective immunity against plague [2]. Recently the plasmid-located gene cafl encoding the capsular antigen $\mathrm{F} 1$ of $Y$. pestis has been cloned and sequenced [3]. The cosmid pl 53 carrying a segment (of about $40 \mathrm{~kb}$ in size) of plasmid pFra mediated the synthesis of the antigen F1, that enabled the detection of the p153 in the library of pFra clones by enzyme immunoassay. At the same time the recombinant plasmid $\mathrm{pF} 18 \mathrm{~L}$ carrying the cafl gene (Fig. 1A) and a fairly long preceding segment (263 bp) involving a possible promoter and ribosome binding site, did not ensure the F1 expression in $E$. coli [3]. These data stimulated us to identify the genetic elements affecting the expression of the F1 and making up a single operon together with the cafl gene.

\section{MATERIALS AND METHODS}

\subsection{Bacterial strains and DNA manipulations}

The $E$. coli strain HB101 was used as transient host for plasmids. Cultures were grown overnight while shaking at $37^{\circ} \mathrm{C}$ in liquid $\mathrm{L}$.B or on solid medium supplemented with $50 \mu 8 / \mathrm{ml}$ ampicillin. Isolation of plasmid DNA by alkali-lysis mellod, DNA cloning and MaxamGilberl sequencing. were performed cssentially as described by

Correspondence address: V.P. Zav'yalov, 142380 Lyubuchany, Moscow Region, Clickhov Distriet, USSR
Maniatis et al. [4]. The plasmid pl2R was used for gene sequencing. Both strands were sequenced.

\subsection{Construction of plasmid derivatives}

Description of plasmids pUC19, pFS2-13, pF18L and cosmids p153, pFS2 was reported earlier $[3,5]$. The cosmid pFS2 was digested with SalGI and then partially with HindIII; $5.7-\mathrm{kb}$ fragment was isolated and cloned into pUC19. The plasmid generated was named p12R. Plasmid p14-34 was constructed by deletion of Pstl-Kpnl fragment of $\mathrm{p} / 2 \mathrm{R}$. Plasmid $\mathrm{NCDB}$ carrying a frame-shift mutation at the unique site BamHI in caflM was constructed by linearizing p14-34 with BamHI, filling in the ends with Klenow fragment and blunt-end ligating.

\subsection{Enzyme immunoassay and immunoblot analysis}

Total F1 content was determined by ELISÁ using peroxidaseconjugated monoclonal antibodies.

Lysates of recombinant $E$. coli strains werc electrophoresed under reducing conditions in $15 \%$ polyacrylamide gel with SDS, and then electroblotted to nitrocellulose. The FI antigen was detected using rabbit polyclonal serum to $\mathrm{Fl}$ and peroxidase-conjugated goat antirabbit lgG.

\section{RESULTS AND DISCUSSION}

To establish the localization of the possible accessory genetic elements in the cafl DNA, we tried to identify the smallest region required for expression of the antigen F1. To achieve this, subclones of p153: pFS2, pFS2-13, p12R, p14-34, pF18L (Fig. 1A) were used ior transformation of the $E$. coli $\mathrm{HB} 101$ and $F 1$ production mediated by the plasmids was analysed by enzyme immunoassay and immunoblot analysis. As shown in Fig. 1B high level of the F1 expression was mediated by plasmids p12R, p14-34 and cosmid pFS2, whereas the shorter plasmids pF18L and pFS2-13 failed to promote 

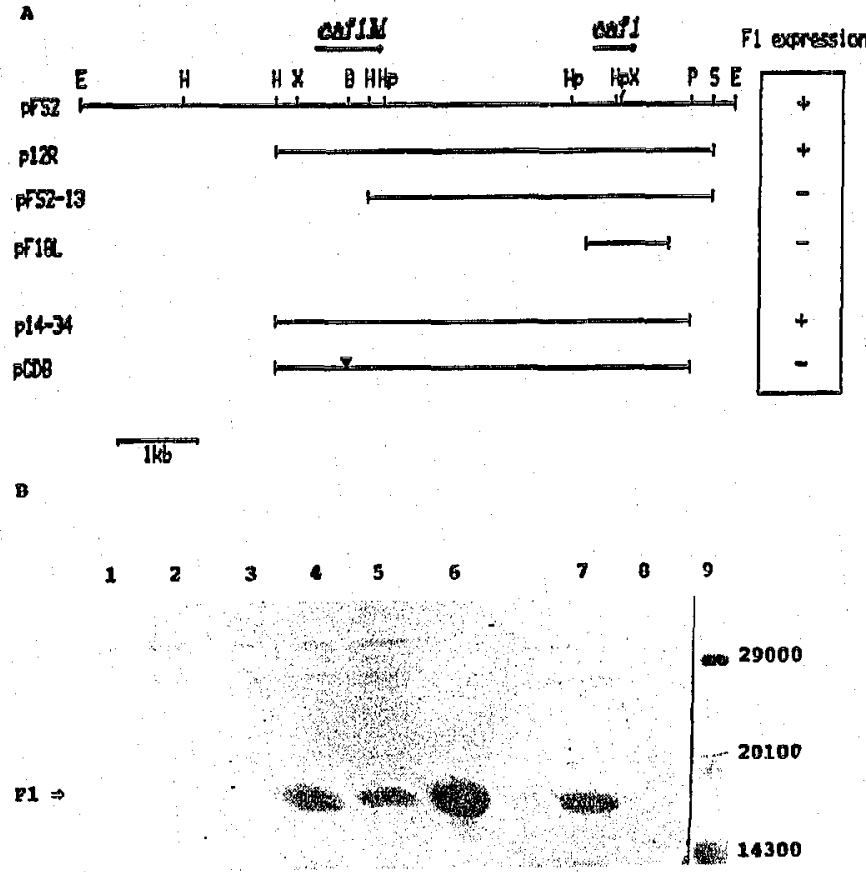

Fig. 1. (A) Restriction map of pFS2 and its derivative plasmids; plasmid-mediated FI expression as estimated by the enzyme immunoassay. Localization of cafl and caflM genes is indicated by arrows at the top. Position of the shift mutation in $\mathrm{pCDB}$ is indicated by $\nabla$. Abbreviations: H, HindIII; Hp, Hpal; P, Pstl; S, SalGI; E, EcoRI; B, BamHI. (B) Immunoblots of the proteins from cell lysates. Lanes: (1) HB101/pUC19; (2) HB101/pF18L; (3) HB101/pFS2-13; (4) HB101/p12R; (5) HB10I/pFS2; (6) F1 isolated from Y. pestis; (7) HB101/p14-34; (8) HBI0l/pCDB; (9) biotinylated molecular weight markers (mwm). The proteins were immunostained with rabbit antiFl serum. Incubation with goat anti-rabbit peroxidase-conjugated IgG was followed by the reaction with diaminobenzidine, Biotinylated mwm were stained with peroxidase-conjugated avidin.

a detectable extent of the F1 synthesis. It follows from the results that the deletion of the $1.2-\mathrm{kb}$ HindIIIHindIII fragment of the plasmid p12R generating the pFS2-13 (Fig. 1A) exerts a dramatic influence on the F1 expression. Thus, at least one of the functionally im. portant (for the Fl expression) regions of Caf 1 DNA is completely or partially located inside the fragment.

The $1.1-\mathrm{kb}$ DNA fragment located between the $X b a 1$ and $H p a l$ sites of the plasmid $\mathrm{p} 12 \mathrm{R}$ was sequenced and found to contain an open reading frame 258 amino acid residues long, capable of encoding the 28764-Da protein (Fig. 2). The gene was named caflM (cafl mediator). The open reading frame is preceded by well conserved Shine-Dalgarno sequence, TAAGGAGGT, located 7 bp before the initiation codon. The region upstream of the ribosome-binding site has putative promoter sequences similar to the $E$. coli consensus seguerices $(-35,-10)[6]$ (Fig. 2). The three-fold repe ed sequence, TTAGCTATTTG $\mathrm{C} / \mathrm{TGCA}$, located upstream of the putative promoter (Fig. 2) might be involved in the regulation of the caflM gene.
TCTAGATAGTGGGCCGGCGCCGGGACTAGCTATITGCGCATACCCAGCAACAGCAATCE 60

TAGCTATTTGTGCACGCGCATCAATATCAAAATTAGCTATTTGCGCAACAAGCAAGTGGA 120

GTGCGCGAAAAGCTAAACTTTGTGTGCATTTTTAAATAAAATTGTTCTCAGTGAGGCTGT 180 $-35$ $-10$ GCTACGgATATAAAAATCCCCTTCATTTGTTACCCACCTTTTTACGCATATCGTCGATAT 24

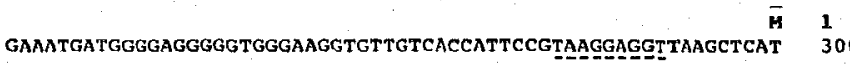

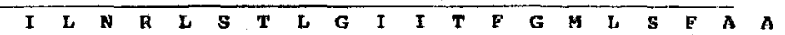
GATTTTAAATAGATTAAGTACGTTAGGAATTATTACTTTCGgCATGCTTAGTTTTGCTGC

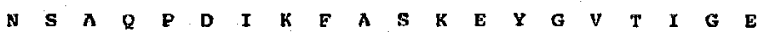
GAACTCTGCTCAACCAGATATCAAATTCGCAAGCAAAGAGTATGGCGTGACTATAGGTGA

S I I I Y GAGTAGgatCATATACCCGTTAGATGCTGCTGGCGITATGGTCTCGgTGaAaAaCACCCA

D Y P V L L I 0 Q AGATTATCCGGTTCTCATTCAGTCTAGGATCTACGACGAGAATAAAGAAAAAGAATCAGA D P $F$ V V $T$ P $P$ L $F$ R L D A GGATCCTTTCGTGGTCACTCCGCCATTGTTTCGATTGGATGCTAAGCAACAAAATTCT'T

R I A $Q$ Q A GCGTATAGCTCAGGCTGGAGGTGTTTTCCCGCGAGATAAAGAGAGCCTAAAGTGGTTATG . 660

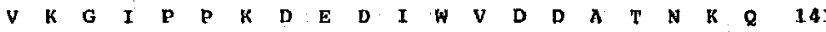

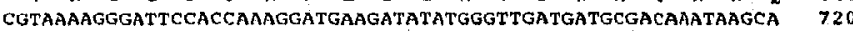

$\begin{array}{lllllllllllllllllllll}K & F & N & P & D & K & D & V & G & V & F & V & Q & F & A & \text { I } & \text { N } & N & \text { C } & \text { I } & 16\end{array}$ AAAATTCAATCCAGACAAAGATGTGGGAGTGTCGTGCAATTCGCAATTAATAATTGCAT

$\begin{array}{lllllllllllllllllllll}\text { K } & \text { L } & \text { L } & \text { V } & \text { R } & \text { P } & \text { N } & \text { E } & \text { L } & \text { K } & \text { G } & \text { T } & \text { P } & \text { I } & \text { Q } & \text { F } & \text { A } & \text { E } & \text { K } & \text { L } & 181\end{array}$ TAAGCTTTTGGTTCGACCGAATGAAITAAAAGGAACCCCTATACAGTTTGCTGAAAAGTT 840

$\begin{array}{lllllllllllllllllllll}S & W & K & V & D & G & G & K & \text { L } & I & \text { A } & E & N & \text { P } & \text { S } & \text { P } & \text { F } & Y & M & N & 201\end{array}$ AAGCTGGAAAGTTGATGGGGGAAGCTAATTGCTGAAAACCCCTCACCTTTCTACATGAA 900

I G E L T F G G K S I P S H Y I P P K S 221

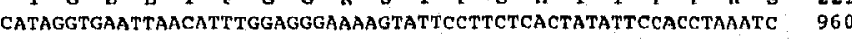

$\begin{array}{lllllllllllllllllllll} & \text { W } & A & F & D & L & P & K & G & L & A & G & A & R & N & V & S & W & R & I & 241\end{array}$ GACGTGGGCTTTTGATTTGCCANAAGgAC'TAGCGgGAGCACGTAATGTTTCGTGGAGAAT' 1020

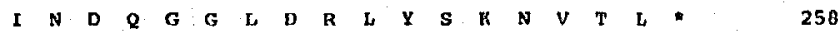
AATIAATGATCAGGGAGGGTTGGATCGT'TTGTATTCCAAAAATGTGACTTTATGATGATG 1080

TभTAAAGGGGACGGGAATAATGAGGTATTCAAAGCTGTTCCTGTGTGCAGGTTAAC

1136

Fig. 2. Nucleotide sequence of the caflM gane and deduced amino acid sequence. The -35 and -10 hexamers of possible promoter are underlined; a probable ribosome-binding site (Shine-Dalgarno sequence) is marked with dashed lines; repeated sequences are boxed.

The line above the sequence indicates the possible signal peptide.

To elucidate the importance of the caflM gene product, we constructed plasmid pCDB with the frameshift mutation in cafl $M$ by inserting additional 4 bp at the unique BamHI site in p14-34. From the DNA sequence of the caflM gene (Fig. 2) the mutated gene was predicted to give rise to a translation product carrying 82 amino acids of the $\mathrm{N}$-terminal portion (including the signal sequence) of the normally 258 amino acids long Caf $1 \mathrm{M}$ followed by 14 erroneous amino acids encoded by the additional 4 bp and the +2 frame. This frameshift mutant unlike the wild-type did not produce the FI antigen detectable by enzyme immunoassay or immunoblot analysis (Fig. IB). The presented data show that the effective synthesis of the capsular antigen Fl of $Y$. pestis is mediated by the expression of the caflM gene.

The PIR protein sequence library was used to search for homology between CaflM and other protein se- 
Caf1M 1MILNRLSTLGI ITFGMLSE-AANSAQPDIKEASKEYGVTIGESRIIXPLDAAGVMVSVKNTQD 62 :: : . : : : :

PapD 1MIRKKILMAAIPLFVISGADA----

A $\quad$ B

-YPVLIQSR I XDENKEKESEDPEVVTPPLFRLDAKQQNSLRIAQAGGV - -F PRDKESLKWLCVKGTPPKD 129

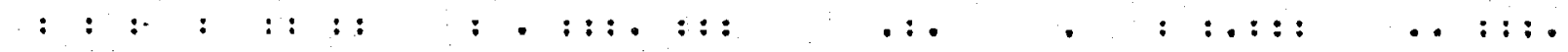

QLPYLAQAWIENENQEK I ITGPVIATPDVQRLDPGAKSMVRLSTTPDISKLPODRESLEYENLREIPPRS 118

$$
\text { C } \mathrm{D}-\frac{\mathrm{E}}{\mathrm{F}}
$$

EDIWVDDATNKQK FNPDKDVGVEVQEAINNCIKLLVRPNELKGTPIQE - AEKLSWKVDGGKLIAENPSP

: :

: : : : : : : : : :

$:: \quad:: \quad: \quad::: \div:$

EKANV- -

EYMN - IGELTFGGKS I PSHYI PPKSTWAEDLPKGLAGARNVSWR I INDQGGLDRLYSKNVTL

: : : $\quad$ : $: \quad: \quad::::: \quad:$

$\frac{\text { YYVTVIGLGGSEKQAE EGEFETVMJSP PRSEQTVKSANYNTPYLSYINDYGGRPVLSFICNGSRCSVKKEK }}{\mathrm{C}} \frac{239}{\mathrm{~F}}$

C

F

$\mathrm{H}$

Fig. 3. Primary structure alignment of the chaperone protein PapD of $E$. coli $[7,8]$ and Caf $1 \mathrm{M}$ of $Y$, pestis, $\beta$-sheet sites in the PapD protein are underlined. The signal peptide sequences are marked with italics.

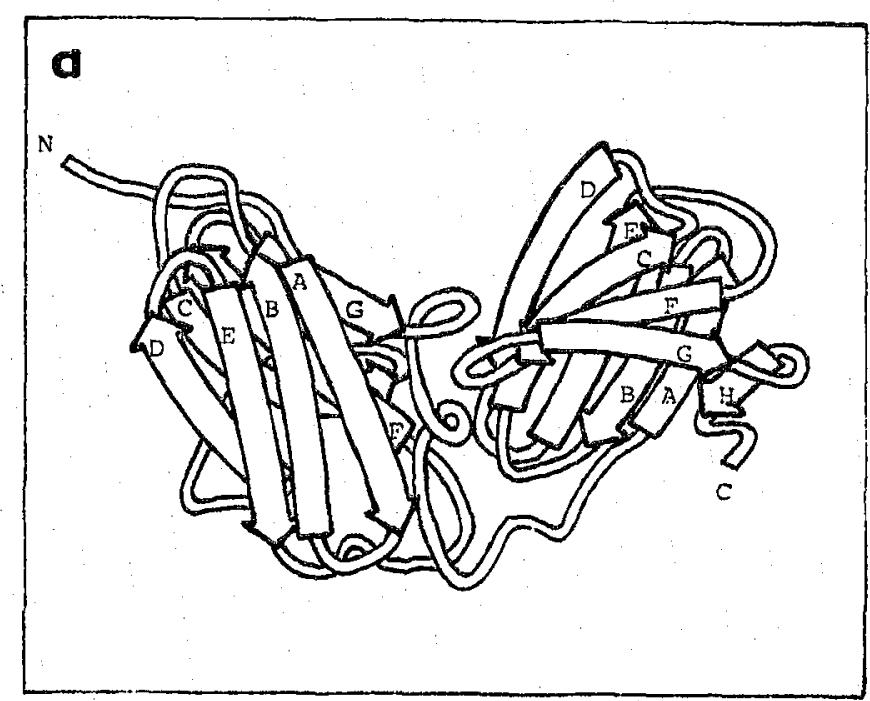

b

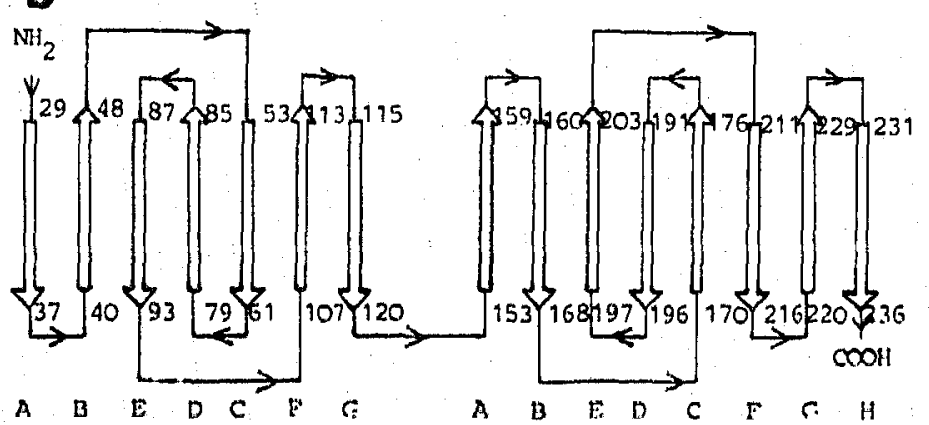

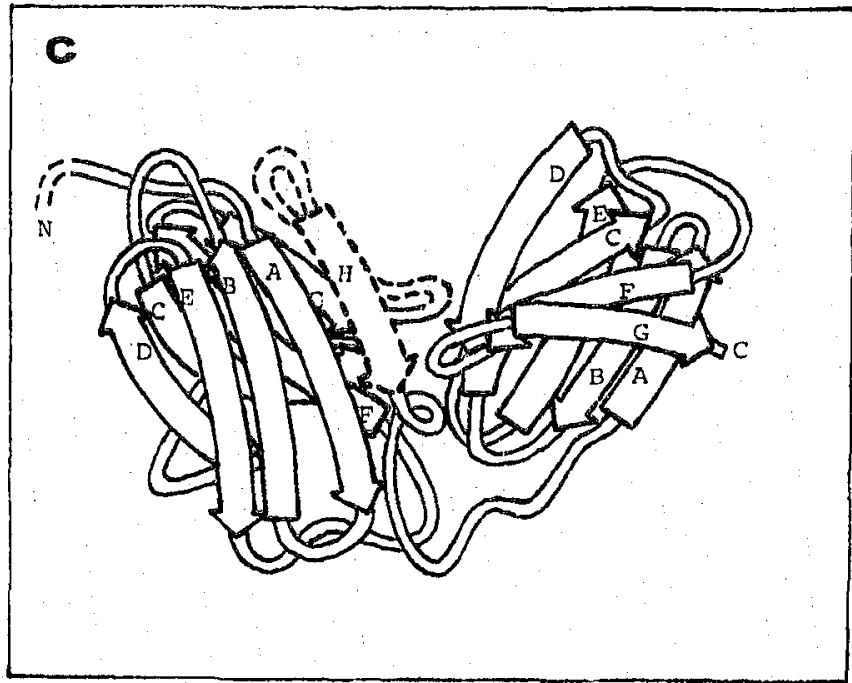

$\mathrm{NH}_{2} \mathrm{~d}$

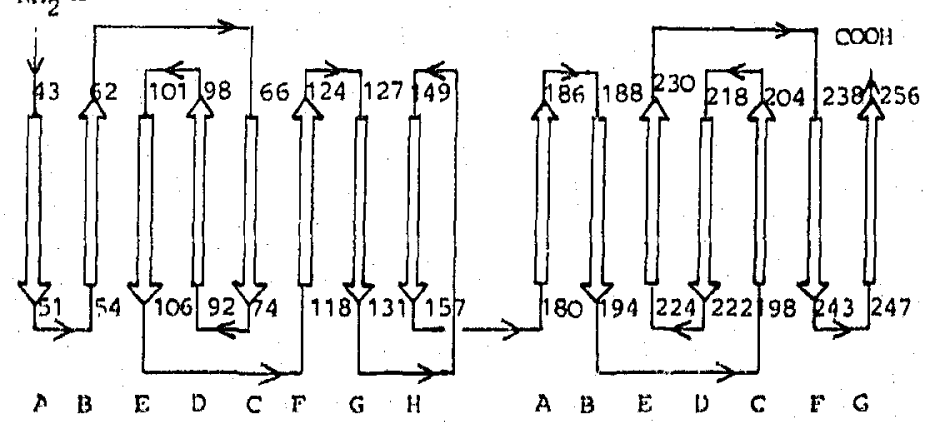

Fig. 4. Tertiary (a) and sceondary (b) structures of the chaperone protein PapD of $E$. coll [8] in comparison with the tentative tertiary (c) and secondary structures (d) of the Carl $M$ protein of $Y$, pestis. Reconstruction of the CariM conformation was carried out using as a basis the tertiary structure of PapD, taking into account primary structures of the proteins compared (Fig. 3). 
quences. The significant homology of $\mathrm{Caf} 1 \mathrm{M}$ with the chaperone protein PapD of uropathogenic $E$. coli $[7,8]$ was found. The PapD is suggested to function in $P$ pilus biogenesis as a periplasmic transport protein [7]. Fig. 3 shows the amino acid sequence alignment of the proteins compared constructed on the basis of the PapD secondary structure [8]. In comparison with the protein $\mathrm{PapD}$, the Caf1M sequence includes an extended insertion at the beginning of the mature protein and also a rather long insertion between the domains. At the same time the Caf $1 M$ lacks the sequence corresponding to the $\beta$-strand $H$ in the second domain of $\mathrm{PapD}$. In the Caf $1 \mathrm{M}$ there is no possibility of formation of a disulfide bridge in the second domain, but this may take place in the first domain between Cys-121 and Cys-160. Taking into account this disulfide bridge and due to the fact that the $\beta$-sheet conformation is predicted for the CafIM sequence $149-157$ by the three methods [9-11], we tried to reconstruct the Caf $1 \mathrm{M}$ spatial structure (Fig. 4c) taking as a basis the known steric structure of PapD [8] (Fig. 4a). As seen, the three-stranded $\beta$-sheet of the first domain making up a tentative binding site of the pili subunits in $\mathrm{PapD}[8]$ is converted into the four-stranded $\beta$-sheet without affecting the general protein architecture and conformation of the hinge region between the domains. In the central part of the putative site of binding of the pili subunits by the PapD protein the solvent-exposed hydrophobic residues V31, L124, 1175, 1215 were replaced by hydrophobic residues $145, V 153,1202,1242$ in the protein CafıM. At the same time the loops forming boundaries of the tentative binding site in the proteins compared significantly differ in their amino acid sequences, which seems to determine specificity of binding of the pili subunits in the case of PapD and F1 antigen in the case of CafiM.
The data presented above and structural resemblance between $\mathrm{Caf} 1 \mathrm{M}$ and $\mathrm{PapD}$ allow one to suggest similar functions of the two proteins. By analogy to the PapD protein $[7,8,12]$, the Caf1M seems to be a periplasmic transport protein and act as a chaperone to promote the posttranslational folding and effective export of components of the $Y$. pestis capsule to the bacterial surface.

Acknowledgements: We are grateful to G.A. Zav'yalova for help in the computer estimations and Dr. P.A. Cherepanov for critical discussion of the work.

\section{REFERENCES}

[1] Protsenko, O.A., Anisimov, P.I., Mozharov, O.T., Konnov, N.P., Popov, Y.A. and Kokushikin, A.M. (1983) Genetika (Moscow) 19, 1081-1091.

[2] Meyer, K.F, Hightower, J.A. and McCrumb, F.R. (1973) J. Infect. Dis. Suppl. 129, 41-45.

[3] Galyov, E.E., Smirnov, O.Yu., Karlishev, A.V., Volkovoy, K.I., Denesyuk, A.l., Nazimov, I.V., Rubtsov, K.S., Abramov, V.M., Dalvadyanz, S.M. and Zav'yalov, V.P. (1990) FEBS l.ett. $277,230-232$.

[4] Maniatis, T., Fritsch, E.F. and Sambrook, J. (1982) Molecular Cloning: A Laboratory Manual, Cold Spring Harbor Laboratory, Cold Spring Harbor, NY.

[5] Yanisch-Perron, C., Vieira, J, and Messing, J. (1985) Gene 33, 103-119.

[6] Rosenberg, M. and Court, D. (1979) Annu. Rev. Genet. 13, 319-353.

[7] Lindberg, F., Tennent, J.M., Hultgren, S.J., Lund, B. and Normark, S. (1989) J. Bacteriol. 171, 6052-6058.

[8] Holmgren, A. and Branden, C.-1. (1989) Nature 342, 248-251.

[9] Zav'yalov, V.P. (1983) Bíochim. Biophys. Acta 310, 70-75.

[10] Novotny, J, and Auffray, C. (1984) Nucleic Acids Res, 12, $243-255$.

[11] Ptitsyn, O.B., Finkelstein, A.V. and Murzin, A.G. (1985) FEBS Lett. $186,143-148$.

[12] Huligren, S.J., Lindberg, F., Magnusson, G., Kihlberg, J., Tennent, J.M. and Normark, S. (1989) Proc. Natl. Acad, Sci. USA 86, 4357-4361. 